\title{
Self-Powered Wireless Vibration-Sensing System for Machining Monitoring
}

\author{
Tien-Kan Chung*a, Hao Lee ${ }^{\mathrm{a}}$, Chia-Yung Tseng ${ }^{\mathrm{a}}$, Wen-Tuan Lo ${ }^{\mathrm{a}}$, Chieh-Min Wang ${ }^{\mathrm{a}}$, \\ Wen-Chin Wang ${ }^{\mathrm{b}}$, Chi-Jen Tu ${ }^{\mathrm{b}}$, Pei-Yuan Tasi ${ }^{\mathrm{b}}$, and Jui-Wen Chang ${ }^{\mathrm{b}}$ \\ ${ }^{a}$ Department of Mechanical Engineering, National Chiao Tung University, Hsinchu 30010, Taiwan. \\ ${ }^{\mathrm{b}}$ Precision Machinery Research and Development Center, Taichung 40768, Taiwan.
}

\begin{abstract}
In this paper, we demonstrate an attachable energy-harvester-powered wireless vibration-sensing module for millingprocess monitoring. The system consists of an electromagnetic energy harvester, MEMS accelerometer, and wireless module. The harvester consisting of an inductance and magnets utilizes the electromagnetic-induction approach to harvest the mechanical energy from the milling process and subsequently convert the mechanical energy to an electrical energy. Furthermore, through an energy-storage/rectification circuit, the harvested energy is capable of steadily powering both the accelerometer and wireless module. Through integrating the harvester, accelerometer, and wireless module, a self-powered wireless vibration-sensing system is achieved. The test result of the system monitoring the milling process shows the system successfully senses the vibration produced from the milling and subsequently transmits the vibration signals to the terminal computer. Through analyzing the vibration data received by the terminal computer, we establish a criterion for reconstructing the status, condition, and operating-sequence of the milling process. The reconstructed status precisely matches the real status of the milling process. That is, the system is capable of demonstrating a real-time monitoring of the milling process.
\end{abstract}

Keywords: energy harvester, electromagnetic, power generator, vibration, machining monitoring, self-powered, wireless sensor

\section{INTRODUCTION}

Recently in the society of wireless sensors network, researchers demonstrated an innovative wireless sensing/monitoring application for machining monitoring [1]. In the application, the wireless sensing system is used to sense, analyze, and monitor the temperature of the milling cutter in the machining process. This real-time monitoring prevents the machining failure caused by over-heating in the process and subsequently enhances the reliability of the machining tool. However, the maintenance becomes difficult due to the battery replacement issue in the case of numerous sensors used in a sensornetwork. To address this issue, an energy-harvester-powered (i.e., toward self-powered) wireless sensing system is a preferred solution. Nowadays, solar cells are comprehensively used as the energy harvesters for powering the wireless sensing system. However, solar-cell-powered wireless sensing system is not suitable for indoor applications, especially inappropriate for monitoring the machines in a factory. Therefore, researchers use vibrational energy harvesters [2] (such as piezoelectric, electromagnetic, and electrostatic energy harvesters) instead of the solar cells to generate power for the indoor applications. However, the circuit for piezoelectric energy harvesters is more difficult than electromagnetic energy harvesters. Thus, electromagnetic energy harvesters are comprehensively utilized by the researchers to power the wireless sensing systems for the indoor applications. That is, more appropriate for monitoring the machines in a factory. More recently, researchers demonstrated an electromagnetic energy harvester powering a wireless sensing system for health monitoring of a spindle [3]. However, both the harvester and wireless sensing system have to be embedded into the spindle of the machine. Consequently, the spindle must be modified (i.e., customized/non-standard spindle). This causes serious issues in design and manufacturing of the customized/non-standard spindle to both spindle- and machinemanufacturers. More seriously, the customized/non-standard spindle may not be fully compatible with the existing machines. This results serious problems in machining. Therefore, an attachable (i.e., nondestructive for the spindle) selfpowered wireless sensing system is needed. Hence, in this paper, we demonstrate an attachable energy-harvesterpowered wireless vibration-sensing system for machining monitoring.

*tkchung@nctu.edu.tw; phone 886-3-5712121 ext. 55116; fax 886-3-572-0634

Sensors and Smart Structures Technologies for Civil, Mechanical, and Aerospace Systems 2013, edited by Jerome Peter Lynch, Chung-Bang Yun, Kon-Well Wang, Proc. of SPIE Vol. 8692,

86922U - ( ) 2013 SPIE · CCC code: 0277-786X/13/\$18 - doi: 10.1117/12.2009435 


\section{DESIGN}

The conceptual approach of the attachable energy-harvester-powered wireless vibration-sensing system used to monitor a milling-process is shown in figure 1. The self-powered system consists of an electromagnetic energy harvester, MEMS accelerometer, and wireless module [4-7]. The harvester consists of a magnet and inductance. The inductance is attached on the machine while several permanent magnets are attached on the spindle of the machine. During milling, the magnets rotate with the spindle while the inductance is fixed on the machine. Due to this, a continuous relative-motion is produced between the rotated magnets and fixed inductance, i.e., the magnets produces a periodic/varied magnetic flux to the inductance. Due to the electromagnetic-induction, the periodic/varied magnetic flux indluces a voltage output in the inductance. That is, a periodic alternating power output is generated. This achieves harvesting mechanical energy from the milling cutter's rotation during the milling process and subsequently converting the mechanical energy to an electrical energy. Furthermore, through an energy-storage/rectification circuit, the harvested electrical energy is rectified in order to steadily power both the MEMS accelerometer and wireless module. This achieves a self-powered wireless vibration sensing system. Meanwhile, vibration occurs while the milling cutter and work piece are physically contacted. The system is able to sense the vibration and transmit the vibration signal to the terminal user (i.e., terminal computer). After the terminal computer received the vibration data, the data are analyzed through a criterion we established in order to indicate the actual status/condition/operating-sequence of the milling. That is, a real-time-monitoring of the milling.

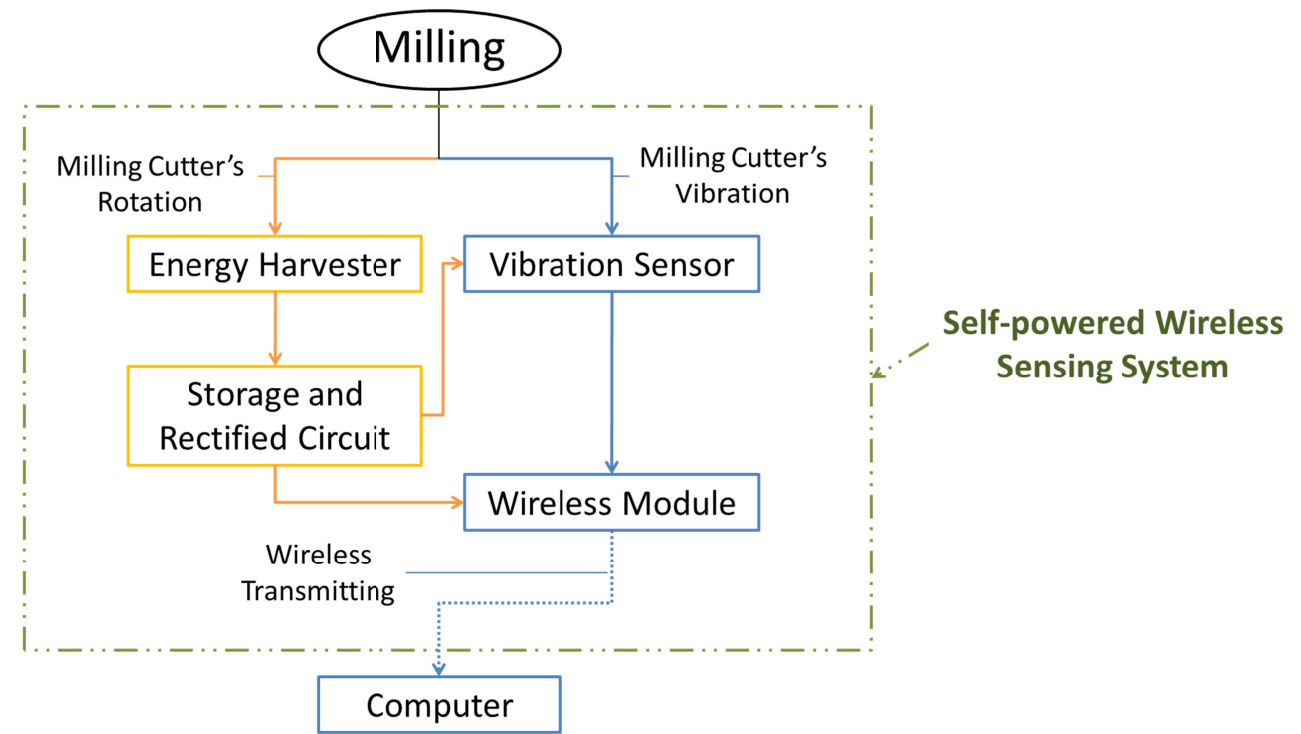

Figure 1. The conceptual approach of the self-powered wireless vibration-sensing system

\section{FABRICATION AND TESTING}

According to the conceptual approach in figure 1, the corresponding practical approach (also referred as an illustration of the test setup) is shown in figure 2. According to the practical approach, the core devices are fabricated and subsequently assembled as the energy-harvester-powered (i.e., self-powered) wireless vibration-sensing system. A photograph of the devices we fabricated for the system is shown in figure 3. The system consists of an electromagnetic energy harvester [shown in figure 3(a)], MEMS accelerometer (i.e., vibration sensor) [shown in figure 3(b)], and wireless module (i.e., a microcontroller/RF-antenna transmitter) [shown in the right side of figure 3(c)]. The electromagnetic energy harvester is fabricated by utilizing an inductance with $\mathrm{NdFeB}$ magnets. The inductance is fixed on the machine while the magnets are attached on the spindle. The energy-storage/rectification circuit of the energy harvester is modified from a bridge-circuit combined with voltage regulator, as shown in the left side of figure 3(c). The MEMS accelerometer we used in the system is Analogy Device, AXDL 330. This accelerometer is sensitive to low-frequency vibration frequently occurred in the machining process. The accelerometer is glued to the over arm of the milling machine in order to experience the vibration generated from the cutting tool while milling. The wireless module utilizes a low-power-consumption ZigBee 
communication-protocol [8-9] based wireless transceiver (Pixie Lite, Flexipanel) with CC2420 ZigBee-ready RF transceiver for wireless data-communication (i.e., transmitting the vibration signal from the module to the terminal computer). A Microchip PIC18F2520 is used in the module for processing signals and controlling the RF transceiver. In order to distinguish the sensor's signal to noise, the output signal of the sensor is amplified by an instrumented amplifier before transmitted to the wireless module. The wireless module is set beside the column of the milling machine to avoid the swarf generated from milling. Finally, through integrating the energy harvester, MEMS accelerometer, and wireless module, an energy-harvester powered wireless vibration-sensing system is achieved and ready for testing.

The illustration and photograph of the testing setup is shown in figure 2 and 4 , respectively. A milling machine shown in figure 4(a) is chosen to be monitored. The testing condition we decided in this paper is described as following. The spindle is rotated with a rotational speed of $1650 \mathrm{rpm}$. The work piece is a SKD-11 tool-steel thick plate. The top and side view of the thick plate is shown in figure 4(b) and 4(c), respectively. An 8-mm tungsten carbide milling cutter is selected as the machining tool shown in figure 4(d).

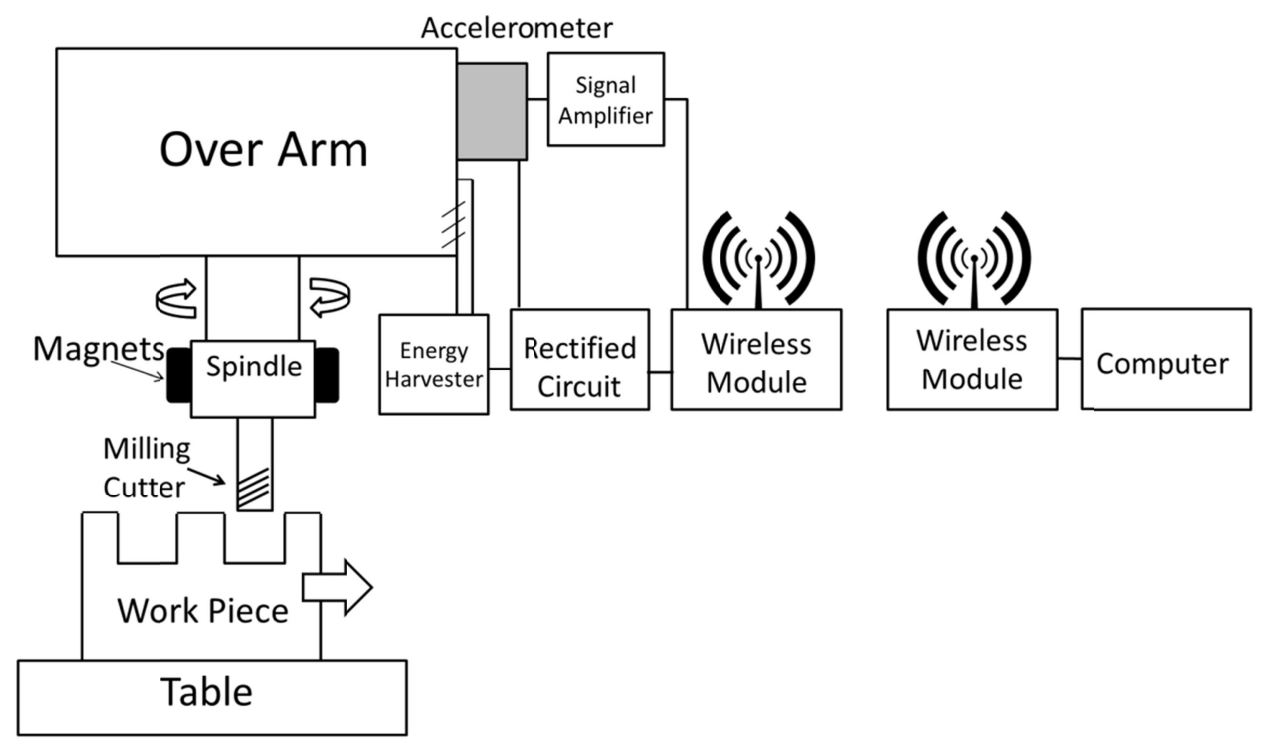

Figure 2. The corresponding practical approach of the self-powered wireless vibration-sensing system monitoring the milling process through sensing/analyzing the milling cutter's vibration (the approach is also referred as an illustration of the test setup).

(a)

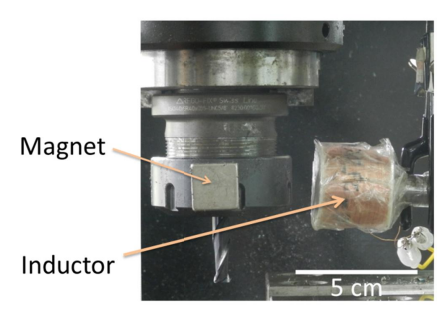

(b)

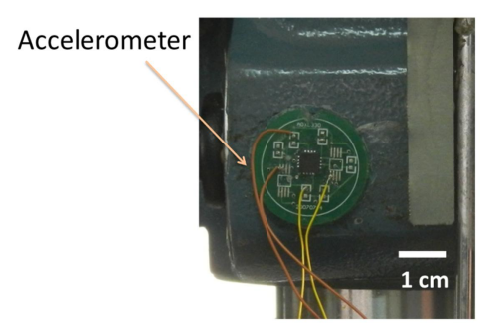

(c)

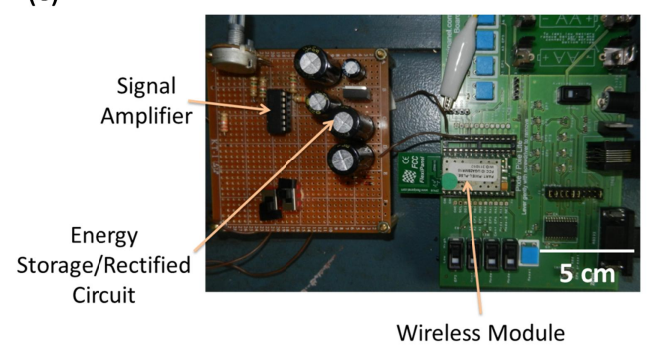

Figure 3. The photograph of the (a) energy harvester, (b) MEMS accelerometer, and (c) wireless module with energy storage/rectified circuit. 
(a)

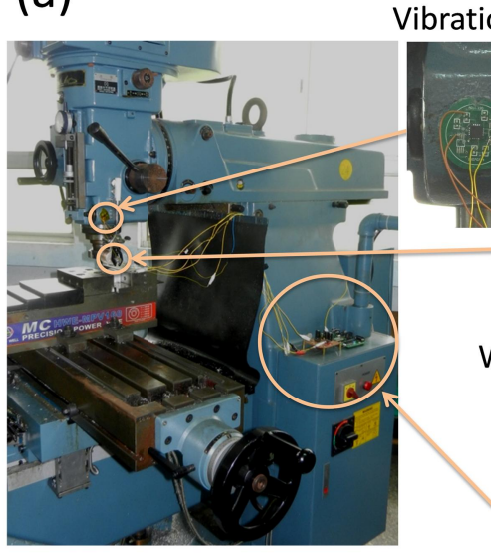

(b)

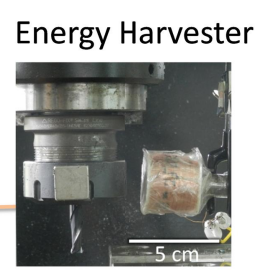

Wireless Module \& Energy Storage/Rectified Circuit

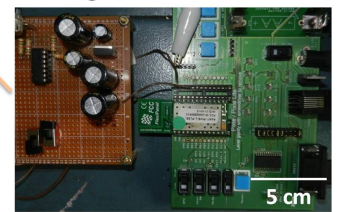

(d) (c)
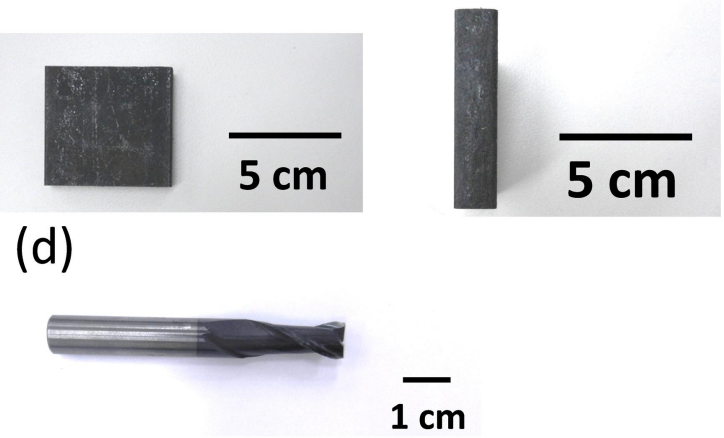

Figure 4. The photograph of (a) the testing setup, (b) front view of the work piece, (c) side view of the work piece, and (d) the milling cutter.

\section{RESULTS AND DISCUSSION}

The test result of the self-powered wireless vibration-sensing system monitoring the milling process (the SKD-11 toolsteel thick plate milled by the 8 -mm tungsten carbide cutter) is shown in figure 5 . In figure 5, the blue solid-line represents a real operating-sequence of the milling (i.e., idle, milling, and then idle; the status is referred to the right axis of the figure). The red dash-line represents the base line of the sensing signal of the capacitive MEMS accelerometer (that is, the base line is corresponded to the bias-voltage for activating the accelerometer). Due to this bias-voltage, the output voltage signals of the accelerometer are oscillated above and below the base line while the accelerometer experiences the vibration produced by milling. Subsequently, the output voltage signals (i.e., vibration data) produced by the accelerometer are transmitted from the system to the terminal computer by the wireless module of the system. Because we set the wireless module to send signals from the system to the terminal computer once per every two seconds, there are total 56 data received by the terminal computer during the milling lasting for 110 seconds. These data received by the terminal computer are fitted by the black curve in the figure. According to the data, line, and curve, the correlation between the operating-sequence of the milling and the output voltage signals (indicating the vibration) received by the terminal computer is obvious. While the cutter mills the work piece, the terminal computer received oscillated voltage signals with a magnitude either higher than $1.3 \mathrm{~V}$ or lower than $1.0 \mathrm{~V}$. This result shows the vibration produced by the milling does oscillate the accelerometer resulting in oscillated output voltage signals. However, not all of the produced output voltage signals are far apart from the base line. Therefore, we use an oscilloscope with a sampling frequency of $2500 \mathrm{~Hz}$ to record/analyze the vibration signals. The analyzed result shows at least $70 \%$ of the signals are out of the range of $1.0 \mathrm{~V}-1.3 \mathrm{~V}$. That is, most voltage signals (at least $70 \%$ of the signals) are capable of indicating the vibration occurred in the milling. That is, the system successfully senses the vibration occurreid in the milling.

Through analyzing the vibration data we obtained, we establish a criterion capable of reconstructing the milling process with different operating-sequence, milling status, and milling conditions (note: this is extremely important toward a realtime monitoring). For the milling process with a typical operating-sequence (i.e., whether milling the work-piece), the flow chart of the criterion is shown in figure 6(a). Based on the experimental data shown in figure 5 , some of the data received while milling have no significant difference with those received while idling. Thus, in this case, using one voltage-amplitude data in figure 5 as a criterion to directly indicate/distinguish the real-time milling status/condition may result in error. As we mentioned in pervious section, the oscillated output voltage signals have at least $70 \%$ of chance capable of indicating the vibration occurred in milling status. To reduce the chance of false-indicating/detection in monitoring, the actual milling sequence/status is indicated by the criterion with a consequent data set (more than just one amplitude data). That is, the self-powered wireless vibration-sensing system determines the status of the milling process is "Stopped" if the terminal computer had received two continuous data which the amplitude of the voltage signal of both data are ranging from $1.0 \mathrm{~V}$ to $1.3 \mathrm{~V}$. Due to this, the probability of false-detection is significantly decreased to $10 \%$. On 
the other hand, because the data far away from the range of $1.0 \mathrm{~V}-1.3 \mathrm{~V}$ only occurred while milling the work piece, the system determines the status of the milling process as "Proceeding" after receiving this sort of data once. Based on the criterion, we successfully reconstruct the milling status/condition/operating-sequence shown in figure 6(b). The comparison of the reconstructed and real milling status/condition/operating-sequence [figure 6(b)] shows the system is capable of demonstrating a real-time monitoring of the milling process.

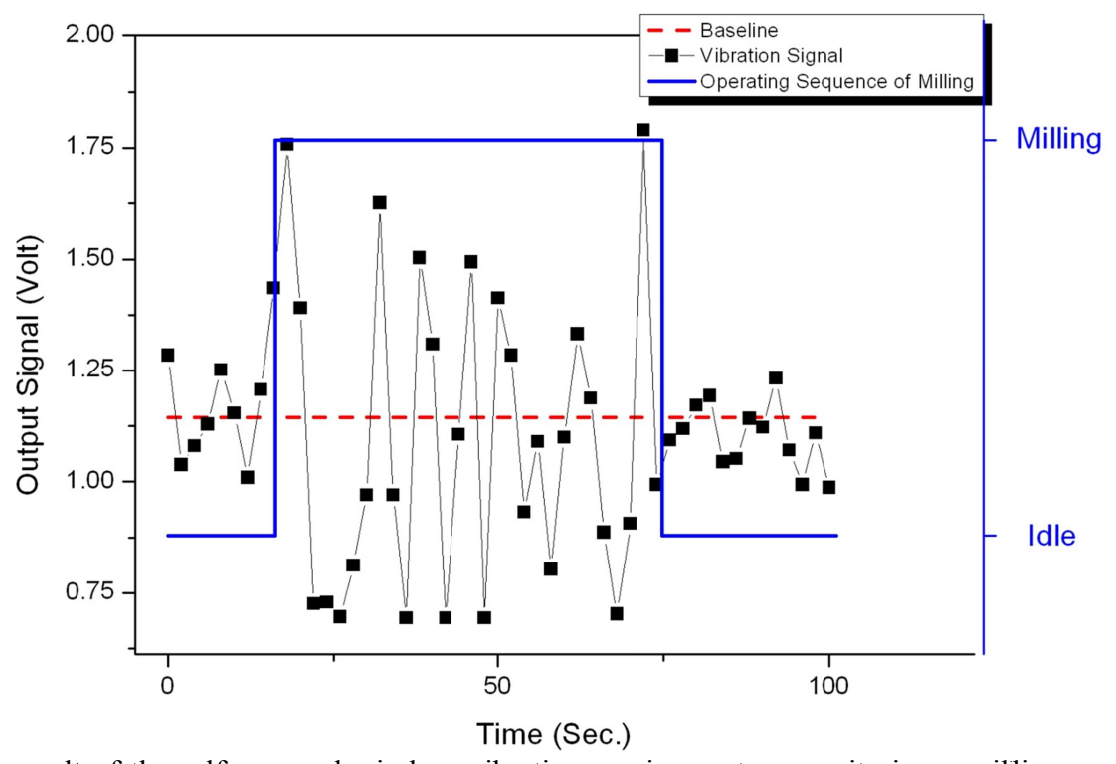

Figure 5. The test result of the self-powered wireless vibration-sensing system monitoring a milling process. The milling cutter and work piece is the 8-mm tungsten carbide cutter and SKD-11 tool-steel thick plate, respectively.

(a)

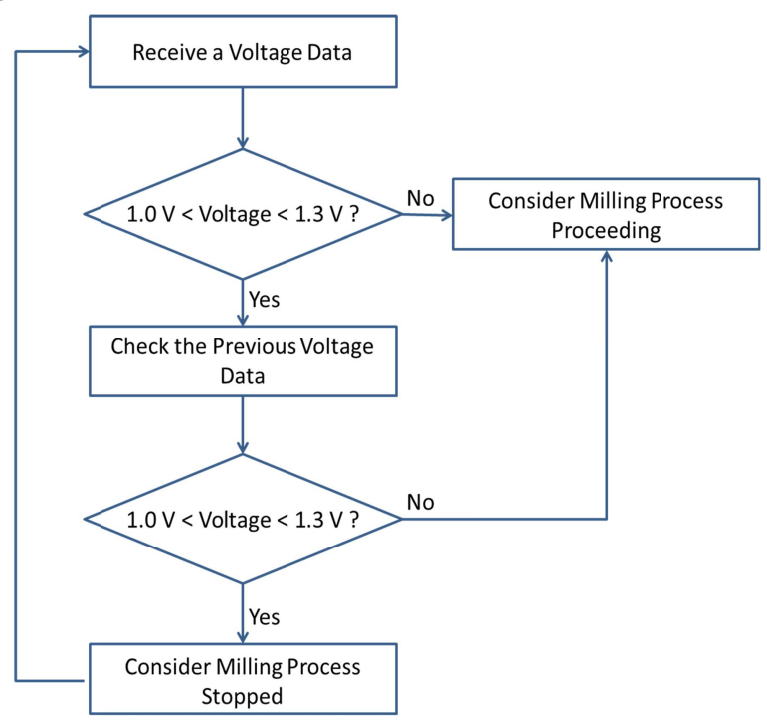

(b)
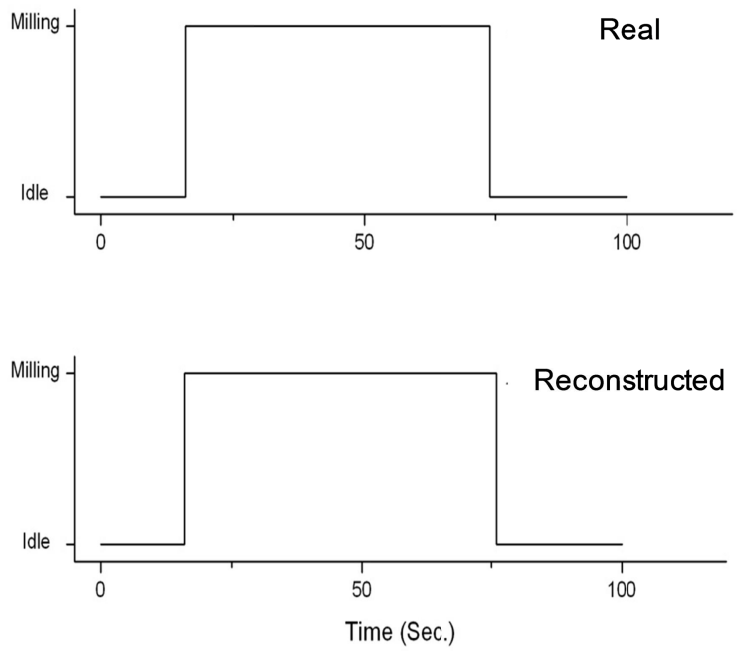

Figure 6. (a) The flow chart of the criterion for indicating the status, condition, and operating-sequence of the milling. (b) The comparison of the real and reconstructed (criterion-based) milling status, condition, and operating-sequence. 


\section{CONCLUSION}

We have successfully demonstrated the energy-harvester-powered (i.e., self-powered) wireless vibration-sensing system for read-time monitoring of a milling-process. The electromagnetic energy harvester harvests the mechanical energy from the milling and subsequently converts the mechanical energy to electrical energy to power the MEMS accelerometer and wireless module. The result shows the system successfully senses the vibration occurred in the milling and subsequently transmits the vibration data (voltage signals) to the terminal computer. Furthermore, based on the criterion we established through analyzing the vibration data received by the terminal computer, the operating-sequence of the milling machine is successfully reconstructed. This achieves a real-time monitoring of the milling process toward an intelligent machining-monitoring system. In the future, we will investigate and develop more advanced smartmachining functions for the self-powered sensing system.

\section{ACKNOWLEDGMENT}

Support for this work was obtained from the Taiwan National Science Council (NSC Grant No.101-2625-M-009-008 and NSC Grant No. 101-2221-E-009-022).

\section{REFERENCES}

[1] Wright, P. K., Dornfeld, D. A., Hillaire, R. G. and Ota, N. K., "A wireless sensor for tool temperature measurement and its integration within a manufacturing system," Transactions of the North American Manufacturing Research Institute of SME. 63-70 (2006).

[2] Chung, T. K., Lee, D. G., Ujihara, M. and Carman, G. P., "Design, simulation, and fabrication of a novel vibration-based magnetic energy harvesting device," Transducers '07 \& Eurosensors XXI, Digest of Technical Papers 1, U438-U439 (2007).

[3] Chang, L. C. and Lee, D. S., "The development of a monitoring system using a wireless and powerless sensing node deployed inside a spindle," Sensors 12(1), 24-41 (2012).

[4] Beeby, S. P., Tudor, M. J. and White, N. M., "Energy harvesting vibration sources for microsystems applications," Measurement Science \& Technology 17(12), R175-R195 (2006).

[5] Lei, W., and Yuan, F. G., "Energy harvesting by magnetostrictive material (MsM) for powering wireless sensors in SHM," Proc. SPIE 6529(2), 652941 (2007).

[6] Beeby, S. P., Torah, R. N., Tudor, M. J., Glynne-Jones, P., O'Donnell, T., Saha, C. R., and Roy, S., "A micro electromagnetic generator for vibration energy harvesting," Journal of Micromechanics and Microengineering 17(7), 1257-1265 (2007).

[7] Kulkarni, S., Koukharenko, E., Torah, R., Tudor, J., Beeby, S., O'Donnell, T. and Roy, S., "Design, fabrication and test of integrated micro-scale vibration-based electromagnetic generator," Sensors and Actuators a-Physical 145-146(1-2), 336-342 (2008).

[8] Yick, J., Mukherjee, B. and Ghosal, D., "Wireless sensor network survey," Computer Networks 52(12), $2292-$ 2330 (2008).

[9] Sung, W. T. and Hsu, Y. C., "Designing an industrial real-time measurement and monitoring system based on embedded system and ZigBee," Expert Systems with Applications 38(4), 4522-4529 (2011). 